\title{
Mitigating Absenteeism in Hospital Workers During a Pandemic
}

\author{
Andrew L. Garrett, MD, MPH, Yoon Soo Park, MS, and Irwin Redlener, MD
}

\section{ABSTRACT}

Objectives: An influenza pandemic, as with any disaster involving contagion or contamination, has the potential to influence the number of health care employees who will report for duty. Our project assessed the uptake of proposed interventions to mitigate absenteeism in hospital workers during a pandemic.

Methods: Focus groups were followed by an Internet-based survey of a convenience sample frame of 17,000 hospital workers across 5 large urban facilities. Employees were asked to select their top barrier to reporting for duty and to score their willingness to work before and after a series of interventions were offered to mitigate it.

Results: Overall, 2864 responses were analyzed. Safety concerns were the most frequently cited top barrier to reporting for work, followed by issues of dependent care and transportation. Significant increases in employee willingness to work scores were observed from mitigation strategies that included preferential access to antiviral medication or personal protective equipment for the employee as well as their immediate family.

Conclusions: The knowledge base on workforce absenteeism during disasters is growing, although in general this issue is underrepresented in emergency planning efforts. Our data suggest that a mitigation strategy that includes options for preferential access to either antiviral therapy, protective equipment, or both for the employee as well as his or her immediate family will have the greatest impact. These findings likely have import for other disasters involving contamination or contagion, and in critical infrastructure sectors beyond health care. $\quad$ (Disaster Med Public Health Preparedness. 2009;3(Suppl 2):S141-S147)

Key Words: disaster, workforce, ability, willingness, absenteeism, hospital, personnel

A $\mathrm{n}$ influenza pandemic, as with any disaster involving contagion or contamination, has the potential to influence the number of health care employees who will report for duty for a variety of reasons. Absenteeism in a disaster is directly influenced by 2 important variables-an employee's personal willingness to accept the risk (real or perceived) of reporting for duty, and the employee's logistical ability to show up for work. Reports from the prehospital, hospital-based, and public health sectors suggest that projected absenteeism rates may be significant during incidents such as a pandemic or severe acute respiratory syndrome (SARS), or in a terrorist incident involving a chemical, biological, radiological, or nuclear agent. In some cases, $50 \%$ or more of respondents indicated either a negative response or uncertainty to the question of whether they would report for duty under these conditions. ${ }^{1-4}$ The issue of worker absenteeism is an underresearched area of disaster medicine. ${ }^{5}$ As a component of the nation's critical infrastructure, the health care system needs to address absenteeism in its disaster plans; however, there is sparse evidence to guide policymakers. Our project evaluated interven- tions intended to mitigate absenteeism in hospital workers and provides initial recommendations to emergency planners in the health care sector.

\section{METHODS}

In 2007 the National Center for Disaster Preparedness at Columbia University's Mailman School of Public Health convened a series of four 90-minute focus groups with employees of a large urban medical center. These moderated sessions consisted of a convenience sample of employees from many areas of the medical center, including clinical (eg, nursing, physicians, dentists), operational (eg, security, administration, facilities), and support (eg, laboratory, blood bank).

Employees were presented with a public health emergency scenario consistent with a "moderate" influenza pandemic as detailed in the third National Planning Scenario, ${ }^{6}$ with widespread cases of illness, a heavy demand for health and public health services, a mounting number of patients requiring critical care, and an increasing number of fatalities. Employees were asked to discuss their ability and willingness to report for duty in the hospital, as well as any issues 
that would stand in the way of doing so. As the moderated discussions evolved, variables were interjected into the discussion (if they did not come up on their own) to explore how they would modify opinion. Examples include a closure of the school system, a reduction in the availability of public transportation, the presence of dependents in the home of the employee (eg, children, elders, pets), or the issue of perceived liability for working under disaster conditions. The sessions were transcribed and analyzed by 2 reviewers to determine which barriers to reporting for duty were the most prevalent, as well to assess any consensus in the opinions of employees on existing or proposed steps to mitigate absenteeism during a disaster.

The findings from these focus group sessions, as well as those from a comprehensive literature analysis, were then used to inform an Internet-based survey. The purpose of this phase of the project was to determine the employee uptake of proposed steps to mitigate absenteeism during a public health emergency. A convenience sample was established consisting of all of the employees of 5 large urban health care facilities in the New York City area. This represented approximately 17,000 employees of 2 university medical centers, a freestanding pediatric hospital, a community hospital, and a behavioral health facility. Recruitment was facilitated by a series of 2 hospital-wide e-mails from the senior administration with a link to the anonymous online survey. Posters were sent to all department heads for posting, color placards were used in public areas of the hospital, and a prominent advertisement was placed on the hospital Intranet. The survey instrument presented the same hypothetical moderate pandemic influenza scenario as the focus groups, and the survey was conducted by the National Center for Disaster Preparedness with the assistance of the administration and emergency planning officials at each facility.

After reading the scenario, participants established a baseline "willingness to work score" (WTWS) using a continuous 0 to 100 scale, with 0 representing "absolutely will not report for duty" and 100 representing "absolutely will report for duty." Participants then selected from a list the most significant barrier that would prevent them from reporting for duty or they could write in a choice. Depending on the barrier they selected, a series of independent interventions was offered to the participants, who rereported their WTWS after each offering. The list of barriers as well as the interventions were established from the focus group findings as well as a review of the literature and current or proposed hospital administrative practice. Interventions were targeted at mitigating issues of both ability and willingness to report for duty. Some general interventions were asked of all respondents (eg, the offer of bonus pay), whereas certain interventions that were intended to mitigate a specific barrier were only offered to those respondents selecting that barrier (eg, the offer of reimbursement for dependent care). Demographic information and other details about the employee's work situation were collected for each participant. The survey instrument was able to be completed on any computer connected to the Internet, including those available in many areas at the participating hospitals.

Both phases of this project were approved by the institutional review boards for the participating facilities. Participants in the focus groups received a small cash payment. Those who completed the survey were given the option of linking to a separate Web site to register for a drawing for an MP3 player or gift certificates to a well-known coffee and tea chain.

\section{RESULTS}

In the 28-day period that the survey was in the field, 2864 responses were received, distributed from among the following self-identified functional job categories: practitioner $19.7 \%$, nursing $29.0 \%$, administration $20.9 \%$, clinical support staff $11.8 \%$, and nonclinical support staff $18.6 \%$. Of the respondents, $75.4 \%$ were female, which approximates the national average of female hospital workers $(80 \%) .^{7}$ The mean initial WTWS was $75.6 \pm 1.1$, with $74.3 \%$ of the employees working a day shift, $4.9 \%$ afternoon/evening, $10.1 \%$ overnight, and $10.7 \%$ a rotating or varied schedule of shifts. In all, $36.1 \%$ identified having a child younger than 18 years in the home, and $37.3 \%$ identified having a spouse or a partner in the home. The frequencies of barriers cited as the single most significant to the employee are reported in Table 1.

Most of the interventions resulted in a statistically significant increase in the WTWS with $P<.05$ using the dependent $t$ test for paired samples (repeated measures) to compare the scores pre- and postintervention (Table 2). In addition, within the results for each barrier, some interventions resulted in a post-WTWS that was significantly higher than others. Table 3 demonstrates the pooled change in WTWS data across all of the barriers for each of the interventions.

\section{TABLE}

\begin{tabular}{|c|c|c|c|}
\hline \multicolumn{4}{|c|}{ Employees' Most Significant Barriers } \\
\hline Rank & Barrier & $\begin{array}{l}\text { Frequency of } \\
\text { Citation }\end{array}$ & $\%$ \\
\hline 1 & Concern for safety of family & 701 & 25.03 \\
\hline 2 & Personal safety concern & 505 & 18.03 \\
\hline 3 & $\begin{array}{l}\text { Dependent child care } \\
\text { needs at home }\end{array}$ & 453 & 16.17 \\
\hline 4 & $\begin{array}{l}\text { "None" selected as most } \\
\text { significant barrier }\end{array}$ & 359 & 12.82 \\
\hline 5 & $\begin{array}{l}\text { Transportation issues } \\
\text { getting to work }\end{array}$ & 301 & 10.75 \\
\hline 6 & $\begin{array}{l}\text { Dependent adult care } \\
\text { needs at home }\end{array}$ & 181 & 6.46 \\
\hline 7 & $\begin{array}{l}\text { Concern about a lack of } \\
\text { specific training }\end{array}$ & 130 & 4.64 \\
\hline 8 & $\begin{array}{l}\text { "Other issues" selected as } \\
\text { most significant barrier }\end{array}$ & 58 & 2.07 \\
\hline 9 & Pet care needs at home & 34 & 1.21 \\
\hline 10 & $\begin{array}{l}\text { Legal concerns about working under } \\
\text { conditions of disaster }\end{array}$ & 11 & 0.39 \\
\hline
\end{tabular}

"Refused" and "don't know" removed ( $n=68,2.43 \%)$. 
TABLE 2

Change in Willingness to Work Score (WTWS) by Barrier and Intervention

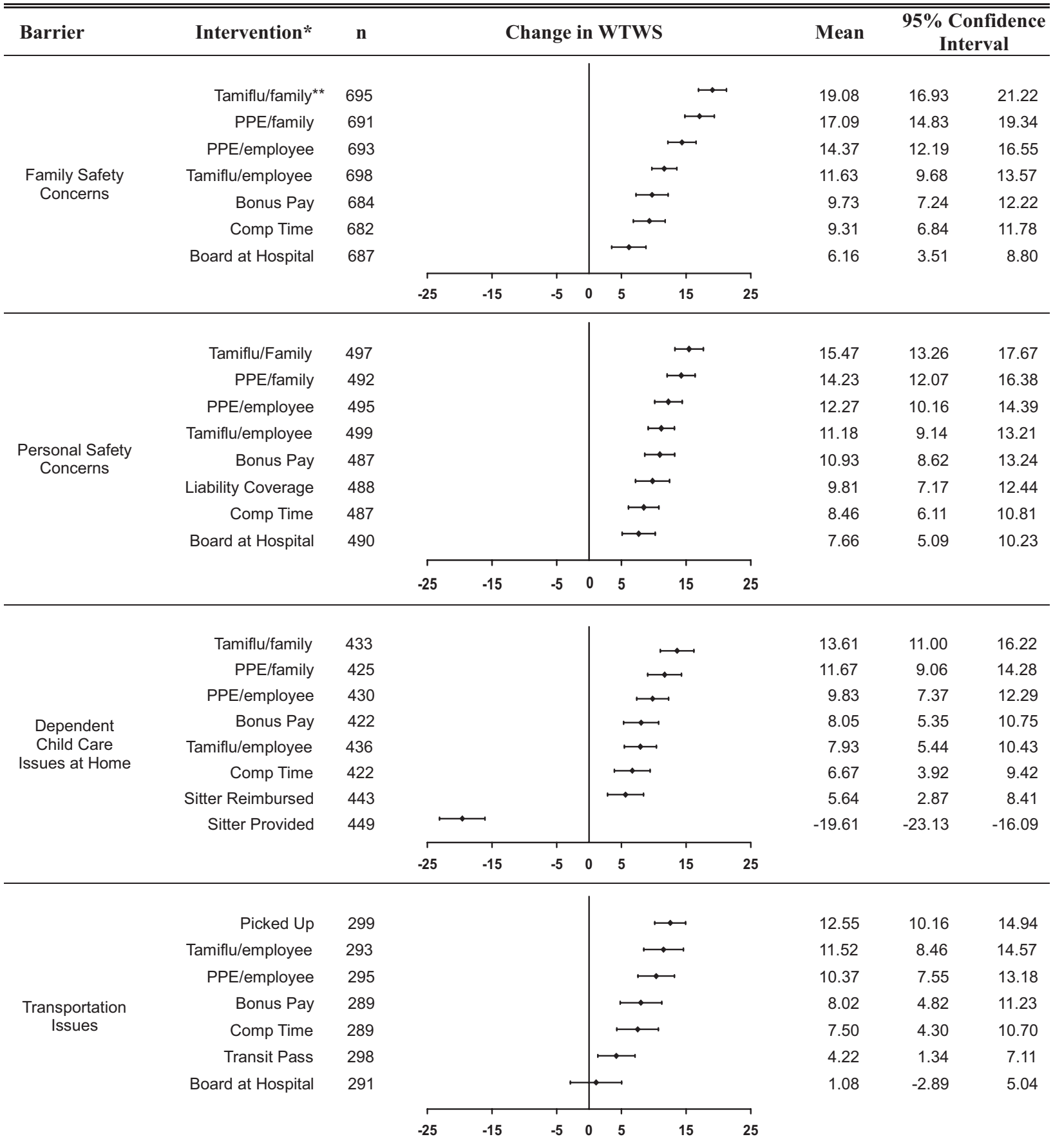

(Continued) 
TABLE 2

Change in Willingness to Work Score (WTWS) by Barrier and Intervention (Continued)

\begin{tabular}{|c|c|c|c|c|c|c|c|c|c|c|c|c|}
\hline Barrier & Intervention* & $\mathbf{n}$ & \multicolumn{7}{|c|}{ Change in WTWS } & Mean & \multicolumn{2}{|c|}{$\begin{array}{c}\text { 95\% Confidence } \\
\text { Interval }\end{array}$} \\
\hline \multirow{9}{*}{$\begin{array}{l}\text { Dependent } \\
\text { Adult Care } \\
\text { Issues at Home }\end{array}$} & PPE/employee & 172 & & & & & & & & 8.79 & -2.05 & 19.63 \\
\hline & Tamiflu/family & 175 & & & & & . & & & 5.38 & 2.02 & 8.74 \\
\hline & PPE/family & 167 & & & & & 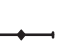 & & & 5.26 & 2.09 & 8.42 \\
\hline & Tamiflu/employee & 176 & & & & & 一 & & & 1.77 & -1.89 & 5.44 \\
\hline & Bonus Pay & 166 & & & & & 一 & & & 1.76 & -1.99 & 5.51 \\
\hline & Comp Time & 166 & & & & & $\rightarrow$ & & & 0.37 & -3.80 & 4.54 \\
\hline & Sitter Reimbursed & 177 & & & & - & & & & -0.54 & -3.88 & 2.80 \\
\hline & Sitter Provided & 179 & & & - & & & & & -7.11 & -11.28 & -2.93 \\
\hline & & & -25 & -15 & -5 & 0 & 5 & 15 & 25 & & & \\
\hline
\end{tabular}

*The interventions included the following: (1) preferential access to Tamiflu being ensured for the employee alone (if medically indicated) (Tamiflu/employee), (2) preferential access to Tamiflu being ensured for the employee plus his or her family (if medically indicated) (Tamiflu/family), (3) mask and gloves (PPE) being provided for the employee while off duty (PPE/employee), (4) PPE being provided for home use by the employee plus his or her family (PPE/family), (5) reimbursement offered at a standard rate for a care provider hired by the employee (sitter reimbursed), (6) provision of a government- or hospital-provided care provider in the employee's home (sitter provided), (7) guaranteed liability coverage for actions that the employee would take on the job during the disaster (liability coverage), (8) the opportunity to stay at the hospital between shifts (board at hospital), (9) transportation services provided by the hospital or government (picked up), (10) special access to mass transit if it were operating at a reduced capacity (transit pass), (11) all participants were offered the intervention of additional vacation or compensation days (comp time), (12) all participants were offered the intervention of additional vacation or compensation days (comp time), and (12) all participants were offered the intervention of a financial bonus for reporting for duty (bonus pay) . PPE $=$ personal protective equipment.

${ }^{* *}$ The $95 \%$ confidence intervals (error bars) for interventions that do not cross the 0 line represent a statistically significant WTWS change with $P<.05$. For each barrier, 95\% confidence intervals that do not overlap with other interventions are significantly different, with $P<.05$. In this example, the WTWS change for Tamiflu/ family was significantly greater than PPE/employee, Tamiflu/employee, and board at hospital.

Comparisons were performed using a $t$ test with Bonferroni adjustment. Table 4 shows a ranked comparison of most significant barriers by sex using a $z$ test for proportional differences.

\section{DISCUSSION}

Recent articles on the topic of employee ability and willingness to work during a disaster have commented on its inherent sociological complexity, ${ }^{1}$ and our results also reflect this aspect.

\section{TABLE 3}

Change in Willingness to Work Score (WTWS) by Intervention Across All Barriers

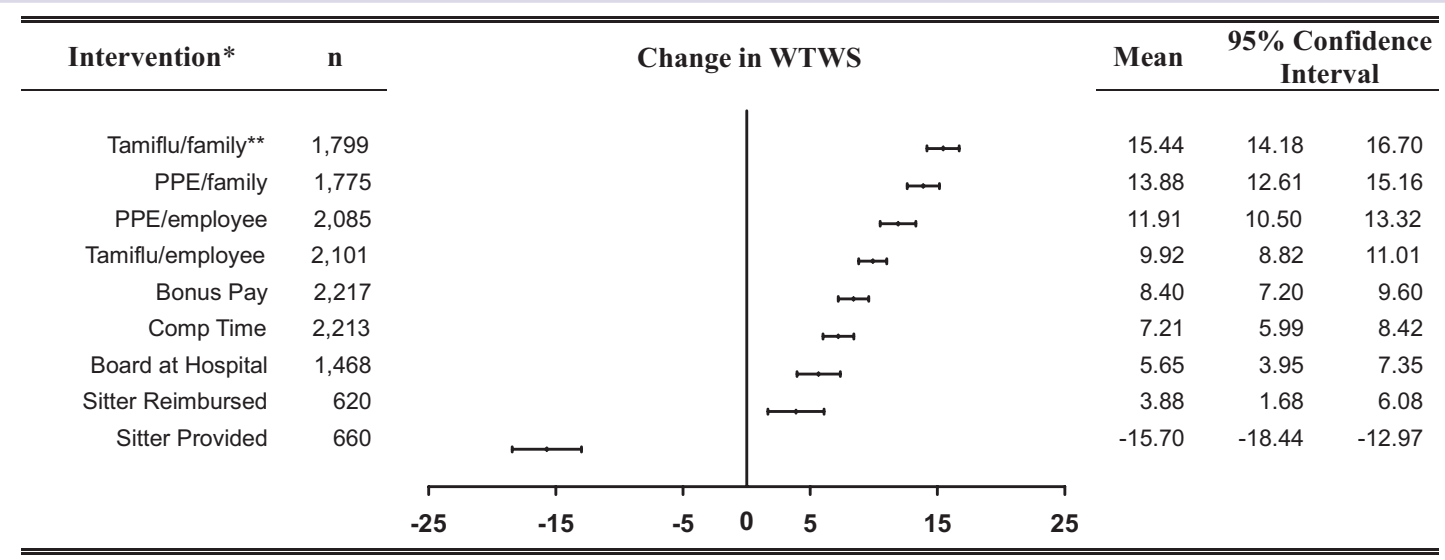

See Table 2 for a description of each intervention.

*95\% confidence intervals (error bars) for interventions that do not cross the 0 line represent a statistically significant WTWS change with $P<.05$.

**95\% confidence intervals (error bars) that do not overlap with other interventions are significantly different with $P<.05$. In this example, the WTWS change for Tamiflu/family was significantly greater than PPE/employee and all interventions listed below it. 


\section{TABIE 4}

\section{Sex Comparison for Most Significant Barrier}

\begin{tabular}{rll} 
Rank & \multicolumn{1}{c}{ Male, $\mathbf{n}=\mathbf{1 9 6 3}(\%)$} & \multicolumn{1}{c}{ Female, $\mathbf{n}=\mathbf{6 3 9}(\%)$} \\
1 & Family safety $(27.39)$ & Family safety $(24.61)$ \\
2 & None identified $(17.68)^{*}$ & Personal safety $(19.41)^{*}$ \\
3 & Personal safety $(15.02)^{*}$ & Child care $(16.96)^{*}$ \\
4 & Child care $(12.36)^{*}$ & None identified $(12.02)^{*}$ \\
5 & Transportation $(11.11)$ & Transportation (10.80) \\
6 & Adult care (6.26) & Adult care $(6.11)$ \\
7 & Lack training (4.69) & Lack training (4.38) \\
8 & Other issues (1.88) & Other issues (2.09) \\
9 & Pet care (0.78) & Pet care $(1.32)$ \\
10 & Legal (0.31) & Legal $(0.31)$ \\
& &
\end{tabular}

"Refused" and "don't know" removed (male $=2.50 \%$, female $=1.99 \%$ ).

*None identified, personal safety, and child care have significant differences in proportion at $P<.05$ using $z$ test for proportions.

An understanding of both the scope and impact of employee absenteeism is still underdeveloped, although a growing literature base clearly demonstrates that this is a variable that must be factored into emergency planning. This is especially true when the hazard includes the risk of contagion or contamination to the employee, as would be seen in an incident involving biological or chemical agents or in a naturally occurring outbreak.

Pandemic influenza was chosen as the hazard for our project (before the 2009 novel H1N1 outbreak) based on both the relative likelihood of an event occurring and previous research that had demonstrated that illnesses such as SARS or pandemic disease would likely have a major impact on workforce absenteeism..$^{3,8}$ Both components of this project attempted to elucidate more information about the intent of hospital workers to report for duty during a pandemic and to provide more details about what types of interventions to mitigate absenteeism may have the highest impact.

In an effort to more quantitatively capture changes in opinion and to reduce the possible effect of central tendency bias, we chose a continuous 0 to 100 scale in lieu of an ordinal Likert scale to capture the WTWS. This facilitated greater precision in the measurement of the pre- and postintervention WTWS change. Our initial mean WTWS of 75.6 suggests that hospital employees in our sample are more likely than not to report for duty before any mitigation efforts. Our results also demonstrate that most of the proposed interventions to reduce absenteeism were likely to increase the employees' WTWS. In the pooled data across all of the barriers, the greatest increase was seen with Tamiflu/family, which resulted in a postintervention WTWS increase of $15.4 \pm 1.3$, to approximately 90.0. The survey instrument implied that in the context of the scenario, Tamiflu would be made preferentially available to the employee and his or her family for use as outbreak prophylaxis and treatment if necessary during the pandemic. We used the brand name Tamiflu (Roche Pharmaceuticals, Nutley, NJ) in both our focus groups and in the survey instrument in lieu of the generic name of the antiviral medication oseltamivir, due to its widespread public recognition. The scenario introduced this topic by the statement, "the antiviral medication Tamiflu may be effective in preventing the illness in those people who take it, and that it may also be effective in treating the flu for those who become sick." The intervention that had the lowest yield overall was offering to provide reimbursement for child care arranged by the employee at a standard rate, with an increase of $3.8 \pm 2.2$. The only intervention that had a significantly negative effect on the WTWS score was offering a free dependent care provider, provided by the hospital or the government and which the employee could not choose, which reduced the WTWS by $15.7 \pm 2.7$. Interventions that included preferential access to Tamiflu or to personal protection equipment (PPE) were the best received. This is promising when one considers that family and personal safety were the 2 most commonly cited top barriers to reporting for work. Employees in this category may be receptive to interventions to increase their willingness to report for work. In addition, it is clear that interventions that included the employee's immediate family in the strategy had a significantly greater impact than those that were intended for the employee alone. The impact of offering additional monetary or time off compensation for working during a disaster was significant, but statistically less so than most of the other options that included Tamiflu or PPE. Offers to the employee to board at the hospital to increase the safety of his or her family through segregation were less effective than most of the other proposals. Dependent care was reported as the third (child care) and fifth (adult care) most significant barrier in our results, but these were the least likely to be effectively mitigated. This may reflect a lack of confidence in the ability of others to provide safe care to a dependent during a pandemic. Interestingly, dependent pet care was cited second to least frequently as the top barrier to reporting for duty. Perhaps employees are more confident in their ability to provide care for their pet while reporting for work, or feel that the risk to their pets is low under these conditions. Transportation issues or concerns was the fourth most commonly cited top barrier; however, employees in this category seem receptive to accepting interventions. Having the hospital provide pickup in a private van or vehicle increased the WTWS by $13.6 \pm 2.4$, suggesting that the concern of being exposed to the public on a train or bus during their commute is an important variable. This concern was also noted in our focus group discussions. Supporting this observation is that the proposed intervention of a "transit pass" designed to facilitate the employee's use of public transportation was less well received, with a WTWS increase of only $4.2 \pm 2.9$. The post-WTWS for private pickup was significantly greater than that for the provision of a special transit pass.

Although the response rate to our survey was relatively low, at approximately $17 \%$, the project benefited from the sample frame size and the large overall number of responses that were 
well distributed among the 5 functional categories of employees. Some previous studies on hospital-based employees explored this issue only in specific categories of clinical workers. ${ }^{1,9,10}$ This study presumes that all hospital employees serve an essential role during a pandemic if the intent is to keep the facility open and secure, the employees paid and on the job to the extent possible, and the full range of health care services maintained during a crisis affecting the community.

Although this project clearly identifies the need for more study, it also provides some useful information that hospital emergency planners can incorporate into current planning. Along with the growing literature base, our data support that an incident such as a pandemic will likely have a significant effect on the number of employees who will report for work. Our results do not answer the question of how many will report versus not report; instead, we provide information about the relative likelihood that an individual employee will choose to report for duty both before and after selected interventions. Because a large number of variables will affect an employee's decision to go to work-such as incidentspecific details from officials and the media, the behavior of other employees, the employee's medical background, and the opinion of family members-it is unlikely that research will be able to prospectively determine with any certainty the magnitude of potential absenteeism for any particular event. Similarly, it is difficult to correlate absenteeism with a reduction in the capability of the hospital system to provide care. Despite this, and especially considering the current climate with the uncertain future of novel H1N1 influenza, the likely most responsible course of action is to mitigate worker absenteeism in the hospital workforce to the extent possible using the best information that is available.

We acknowledge that hospitals and the government have limited options and funding available to them to achieve this goal. Based on the findings of this project and a literature analysis, as well as anecdotal information from the ongoing novel H1N1 outbreak, it is clear that antiviral therapy will be in high demand from employees, patients, and the public. Although a broad chemoprophylaxis strategy for health care workers has not been used in the novel H1N1 outbreak to date, it is an option that may be critical in a more serious pandemic. It is essential that hospital emergency planners appreciate the scope of role that medications such as antivirals and PPE likely will play during a serious pandemic to maximize the number of employees who will both report for work and subsequently stay on the job. Our data suggest that a strategy of ensuring preferential access to antiviral therapy or PPE to both the employee and his or her immediate family will have the highest reduction of absenteeism when compared with other candidate interventions targeted at the employee alone. The findings also suggest that some types of barriers are more amenable to mitigation than others. For example, employees who choose not to report for duty because of concerns about dependent care have lower postWTWS scores for the same intervention than those who are concerned about the safety of themselves or their family. There was clearly a sex effect in the data that is worth considering. Our findings suggest that females may think of personal and family safety, as well as the need for child care, as a greater barrier to work in a pandemic setting than males. Males were more likely to report that they did not have a top barrier preventing them from reporting for duty. Considering that some categories of hospital workers are predominantly female, such as the nursing workforce at $90.9 \%,{ }^{11}$ it may be helpful to prioritize interventions that engage these findings. Taken together, the project results suggest that it would be valuable for the administration of any hospital to better understand the barriers faced by its own employees, something that could be accomplished through local survey work or focus groups. This would enable the administration and planners to develop a customized absenteeism mitigation strategy appropriate for their own environment and budget. Because disasters are a local emergency first, a combined mitigation strategy that includes focused hospital-based efforts complemented by regional, state, and federal assistance is important to foster.

There are limitations to this type of project that include selection bias in that the survey was offered exclusively online to a convenience sample. Reporting bias was also possible because this was a self-administered questionnaire. A multimedia recruitment strategy, the broad availability of Internet-connected computers in the workplace, and the assurance of anonymity to responders were used to minimize these effects. Because we only considered the most significant barrier to the employee, it is possible that the importance of some issues, such as pet care, was not captured in the correct context. Further research should expand this methodology to assess the potentially broad spectrum of barriers that an employee would face in addition to the one that is the single most significant. This project was conducted in a large urban area and as such the findings may not be easily generalizable to other settings. Focus groups and the survey instrument were conducted in English only, which could represent a language bias. The majority of respondents were workers on the day shift, which raises the concern that they may have overrepresented issues such as child care; however, the proportion of respondents stating that they had a dependent child in the home was not significantly different between the shifts. Finally, the use of the WTWS approach has not been validated under real-world conditions of a pandemic - it is uncertain what the realistic effect of a larger versus a smaller change in the WTWS translates to in terms of actual rates of reporting for work.

Although the concept of preferentially providing supplies such as antiviral medications to an employee and his or her family seems to be an effective one in an attempt to maximize the hospital workforce, it is important to place this finding in context. It would not be appropriate to advocate the use of antiviral medication outside of the recommendations of public health authorities. In our "moderate" pandemic scenario, 
it was implied that there were inadequate supplies of Tamiflu for the public to obtain it when they wanted it, regardless whether for outbreak prophylaxis, postexposure prophylaxis, or treatment (similar to the conditions that were seen in some areas during the spring peak of the 2009 H1N1 outbreak). The intervention was intended to reassure an employee (and his or her family) that if it were medically indicated that they take antiviral medications, that they would have preferential access, whether for prophylaxis or treatment. Although we used the antiviral medication oseltamivir (Tamiflu) in our proposed intervention because of its broad public recognition, the antiviral medication zanamivir (Relenza) is also used for pandemic influenza preparedness in much the same context.

\section{CONCLUSIONS}

The knowledge base on anticipating and mitigating workforce absenteeism during certain types of disasters involving contagion or contamination is growing, although in general this issue is underrepresented in the emergency planning efforts of the health and public health sectors, as well as in the rest of the nation's critical infrastructure systems. Our project evaluated the uptake of interventions to mitigate absenteeism in hospital workers during a pandemic. It is uncertain at this time what the true impact of absenteeism will be on the ability of a hospital to deliver care during a pandemic; however, one can be reasonably sure that reducing absenteeism will be an important consideration as part of the overall strategy for hospital resilience. Our data suggest that a mitigation strategy that includes options for either preferential access to antiviral therapy, PPE, or both for the employee as well as his or her immediate family will have the greatest impact, although most of the interventions we explored did significantly increase the WTWS of the employee compared with the baseline.

This project looked specifically at pandemic influenza as the hazard; however, it is likely that the findings elucidated in this report would be useful for other hazards such as other biological agents, either naturally occurring or stemming from a terrorist event, and chemical, nuclear, or radiological hazards. The findings may also have import in critical infrastructure sectors well beyond health care.

\section{About the Authors}

All of the authors are with the National Center for Disaster Preparedness at the Columbia University Mailman School of Public Health.

Address correspondence and reprint requests to Andrew L. Garrett, MD, MPH, National Center for Disaster Preparedness, Columbia University, Mailman
School of Public Health, 215 W 125th St, Suite 303, New York, NY 10027 (e-mail:ag2612@columbia.edu).

The study was supported, in part, by grant 5U90TP224241 from the Centers for Disease Control and Prevention.

Received for publication June 19, 2009; accepted September 9, 2009.

\section{Authors' Disclosures}

The authors report no conflicts of interest.

\section{Acknowledgments}

The authors acknowledge the contributions of Philip Graham, MD, MSc, Jessica M. Prata, Robert B. Bristow, MD, and Carol LeMay of NewYorkPresbyterian Hospital, who assisted with data collection for the survey; David M. Abramson, PhD, MPH, and Tasha Stehling-Ariza, MPH, of Columbia University, who assisted with the concept development and review. Special appreciation goes to Kimberly Gill, MA, and Elisa Ignatius, $\mathrm{BA}$, for their significant early work with the project.

ISSN: 1935-7893 C 2009 by the American Medical Association and Lippincott Williams \& Wilkins.

\section{DOI: 10.1097/DMP.0b013e3181c12959}

\section{REFERENCES}

1. Chaffee M. Willingness of health care personnel to work in a disaster: an integrative review of the literature. Disaster Med Public Health Preparedness. 2009;3:42-56.

2. Irvin CB, Cindrich L, Patterson W, et al. Survey of hospital healthcare personnel response during a potential avian influenza pandemic: will they come to work? Prehosp Disaster Med. 2008;23:328-335.

3. Qureshi K, Gershon RR, Sherman MF, et al. Health care workers' ability and willingness to report to duty during catastrophic disasters. J Urban Health. 2005;82:378-388.

4. Young CF, Persell DJ. Biological, chemical, and nuclear terrorism readiness: major concerns and preparedness of future nurses. Disaster Manag Response. 2004;2:109-114.

5. Abramson DM, Morse SS, Garrett AL, et al. Public health disaster research: surveying the field, defining its future. Disaster Med Public Health Preparedness. 2007;1:57-62.

6. Howe D. National Planning Scenarios. April 2005. http://media.washingtonpost. $\mathrm{com} / w p$-srv/nation/nationalsecurity/earlywarning/NationalPlanningScenarios April2005.pdf. Accessed July 17, 2006.

7. NIOSH Safety and Health Care Topic: Healthcare Workers. http:// www.cdc.gov/niosh/topics/healthcare/. Accessed September 2, 2009.

8. Balicer RD, Omer SB, Barnett DJ, et al. Survey of local public health workers' perceptions toward responding to an influenza pandemic. J Healthc Prot Manage. 2006;22:1-14.

9. Hogg W, Huston P, Martin C, et al. Enhancing public health response to respiratory epidemics: are family physicians ready and willing to help? Can Fam Phys. 2006;52:1254-1260.

10. Lanzilotti SS, Galanis D, Leoni N, et al. Hawaii medical professionals assessment. Hawaii Med J. 2002;61:162-173.

11. Committee on Health, Education, Labor, and Pensions, US Senate. Nursing Workforce: Recruitment and Retention of Nurses and Nurse Aides Is a Growing Concern. Washington, DC: US General Accounting Office; 2001:22. 